\title{
Cardiac T1 mapping in congenital heart disease: bolus versus infusion protocol for measurement of myocardial extracellular volume
}

\author{
Nadya Al-Wakeel", Sanaz Rastin, Frédéric H Münch, Felix Berger, Titus Kuehne, Daniel Messroghli \\ From 18th Annual SCMR Scientific Sessions \\ Nice, France. 4-7 February 2015
}

\section{Background}

Measurement of myocardial extracellular volume fraction (ECV) with T1 mapping cardiac magnetic resonance (CMR) before and after the application of a gadolinium-based extracellular contrast agent enables the assessment of diffuse myocardial fibrosis. The equilibrium between blood and myocardium contrast concentration required for ECV measurements can be achieved with a primed contrast infusion (equilibrium contrastCMR). In healthy volunteers it could be shown that a single bolus may also be sufficient to reach equilibrium. We hypothesized that equilibrium between blood and myocardium contrast distribution can be achieved with the bolus-only technique to accurately measure diffuse myocardial fibrosis in patients with congenital heart disease (CHD).

\section{Methods}

A study group of 23 patients with CHD (age range $14-45$ years, mean age 25.8 years) was compared with 17 healthy volunteers (age range 23 - 30 years; mean age 25.1 years). Using modified Look-Locker inversion recovery (MOLLI) T1 mapping before application, 15 minutes after bolus injection, and during constant infusion of Gd-DOTA, T1 values were obtained for blood pool and myocardium of the interventricular septum (IVS), the left ventricular (LV) antero-/lateral wall, and the inferior or anterior wall of the right ventricle $(\mathrm{RV})$ in a single midventricular plane in short axis or in transverse orientation.

\section{Results}

In CHD patients, correlation between ECV by bolus-only and infusion was strong for IVS, LV and RV $(R 2=0.88$,
0.85 , and 0.79 , respectively). ECV of IVS, LV and RV by both techniques correlated strongly in healthy volunteers $(\mathrm{R} 2=0.91,0.81$, and 0.74 . respectively). Bland-Altman plots did not show significant bias between the techniques in patients and healthy volunteers for any of the analyzed regions (Figure 1).

\section{Conclusions}

Based on T1 mapping, ECV of LV and RV myocardium can be measured accurately by the bolus-only technique in patients with CHD. The use of a bolus-only approach facilitates the integration of ECV measurements into clinical CMR routine workflow across a wide range of CHD.

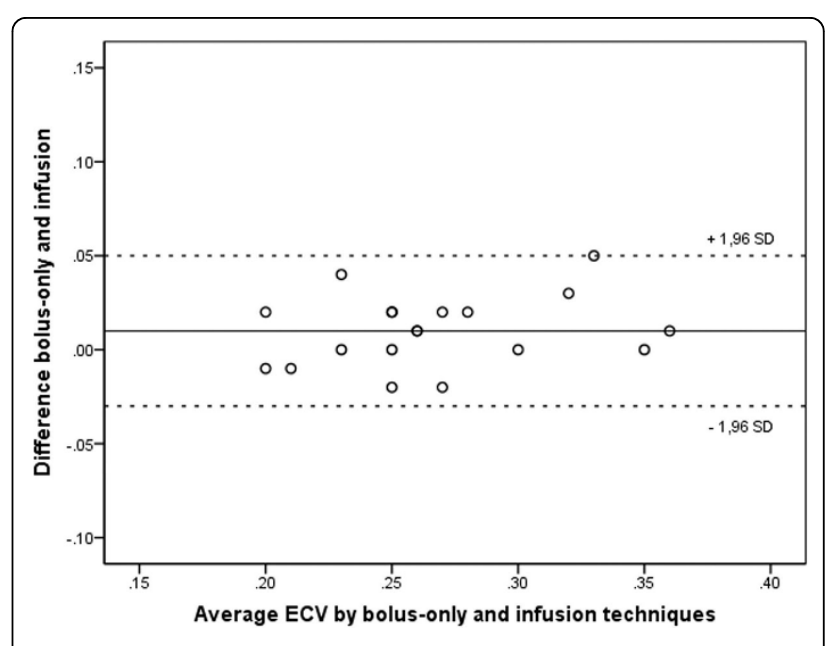

Figure 1 Bland-Altman analysis of ECV by bolus-only and infusion techniques in patients with CHD (LV in transverse orientation)

German Heart Institute Berlin, Berlin, Germany 


\section{Funding}

The study was funded by the German Research Foundation (DFG ME 3508/4).

Published: 3 February 2015

doi:10.1186/1532-429X-17-S1-W22

Cite this article as: Al-Wakeel et al: Cardiac T1 mapping in congenital

heart disease: bolus versus infusion protocol for measurement of

myocardial extracellular volume. Journal of Cardiovascular Magnetic

Resonance 2015 17(Suppl 1):W22.

Submit your next manuscript to BioMed Central and take full advantage of:

- Convenient online submission

- Thorough peer review

- No space constraints or color figure charges

- Immediate publication on acceptance

- Inclusion in PubMed, CAS, Scopus and Google Scholar

- Research which is freely available for redistribution

Submit your manuscript at www.biomedcentral.com/submit
C Biomed Central 